Article

\title{
Theoretical Analysis of Elastic, Mechanical and Phonon Properties of Wurtzite Zinc Sulfide under Pressure
}

\author{
Melek Güler * and Emre Güler \\ Department of Physics, Hitit University, 19030 Corum, Turkey; eguler71@gmail.com \\ * Correspondence: mlkgnr@gmail.com; Tel.: +90-3642277000; Fax: +90-3642277005 \\ Academic Editor: Matthias Weil \\ Received: 18 April 2017; Accepted: 31 May 2017; Published: 4 June 2017
}

\begin{abstract}
We report for the first time the application of a mixed-type interatomic potential to determine the high-pressure elastic, mechanical, and phonon properties of wurtzite zinc sulfide (WZ-ZnS) with geometry optimization calculations under pressures up to $12 \mathrm{GPa}$. Pressure dependency of typical elastic constants, bulk, shear, and Young moduli, both longitudinal and shear wave elastic wave velocities, stability, as well as phonon dispersions and corresponding phonon density of states of WZ-ZnS were surveyed. Our results for the ground state elastic and mechanical quantities of WZ-ZnS are about experiments and better than those of some published theoretical data. Obtained phonon-related results are also satisfactory when compared with experiments and other theoretical findings.
\end{abstract}

Keywords: ZnS; wurtzite; elastic; mechanical; phonon

\section{Introduction}

The computational modeling of materials has been a successful and rapid tool to address the unclear points of physical interest. In addition, predicting good elastic and thermodynamic properties of materials is a necessary demand for present-day solid state science and industry. In particular, these properties at high pressure and temperature are important for the development of modern technologies [1-5].

As emphasized in the authors' recent work, wide-band-gap II-VI semiconductors such as ZnX $(\mathrm{X}: \mathrm{S}, \mathrm{O}, \mathrm{Se}, \mathrm{Te})$ and $\mathrm{CdX}(\mathrm{X}: \mathrm{S}, \mathrm{Se}, \mathrm{Te})$ are remarkable materials for the design of high-performance opto-electronic devices including light-emitting diodes and laser diodes in the blue or ultraviolet region [6].

According to its crystallographic structure, $\mathrm{ZnS}$ compounds can crystallize in either zinc blende (ZB) crystal structure with space group F-43m or wurtzite (WZ) crystal structure with the $\mathrm{P}_{3} \mathrm{mc}_{\mathrm{m}}$ space group at ambient conditions [7-10].

To date, there have been a number of studies performed especially for the ground state $(\mathrm{T}=0 \mathrm{~K}$ and $\mathrm{P}=0 \mathrm{GPa}$ ) structural, elastic, thermodynamic, and optical properties of ZB and WZ crystal structures of ZnS compounds-not only with experiments, but also with theoretical works because of their technological importance. In 2004, Wright and Gale [11] reported new interatomic potentials to model the structures and stabilities of the ZB and WZ ZnS phases in which their theoretical results are within experiments. Later, in 2008, Bilge et al. [8], performed ab initio calculations based on projector augmented wave pseudo potential (PAW). They employed generalized gradient approximation (GGA) of density functional theory (DFT) to investigate the ground state mechanical and elastic properties of $\mathrm{ZB}$, rock salt $(\mathrm{NaCl})$, and $\mathrm{WZ}$ phases of $\mathrm{ZnS}$. They concluded that the mechanical properties of $\mathrm{ZnS}$ under high pressure are quite different from those at ambient conditions. At the 
same time, Rong et al. [10] reported the pressure dependence of the elastic properties of ZB and WZ crystals of ZnS by the GGA within the plane-wave pseudopotential (PWP) of DFT and found reasonable results which are consistent with former experimental and theoretical data. Further, in 2009, Cheng et al. [12] conducted an experimental and theoretical study on first and second-order Raman scattering of ZB and WZ ZnS and found satisfactory experimental and theoretical results. Afterwards, in 2012, Grünwald et al. [9] established transferable pair potentials for ZnS crystals to accurately describe the ground state properties of $\mathrm{ZB}$ and $\mathrm{WZ}$ phases of $\mathrm{ZnS}$ compounds. Recently, Yu et al. [13] performed DFT calculations by using both the local density approximation (LDA) and GGA for the exchange-correlation potential and calculated the phonon dispersion curves and the phonon density of states of WZ ZnS in which the calculated values display good agreement with earlier experimental and theoretical data. In another recent study, Ferahtia et al. [14] published the first-principles plane-wave-based pseudopotential method calculations of the structural, elastic, and piezoelectric parameters of WZ ZnS and concluded a reasonable degree of agreement between their results and data available in the literature.

The above recent and increasing theoretical efforts on $\mathrm{ZnS}$ motivated us to perform this work by addressing the lesser-known high-pressure elastic, mechanical, and phonon properties of WZ ZnS with a different method. In contrast to the above applied methods and interatomic potentials of literature, this is the first report regarding the application of a mixed-type potential to determine the mentioned high-pressure quantities of WZ ZnS with geometry optimization calculations. During calculations, we concentrated on the pressure behavior of five independent elastic constants, bulk, shear, and Young moduli, elastic wave velocities, mechanical stability conditions, and phonon properties of WZ ZnS under pressures between $0 \mathrm{GPa}$ and $12 \mathrm{GPa}$ at $\mathrm{T}=0 \mathrm{~K}$.

The following part of the article provides details of our theoretical calculations with the employed interatomic potential in Section 2. In Section 3, we compare our results with the available experimental results and other theoretical data. Finally, Section 4 presents the main findings of this work in the conclusions.

\section{Details of Theoretical Calculations}

The most significant feature of materials modeling is the choice of the proper interatomic potentials that sufficiently and accurately describe the physical properties of the concerned problem. Simple empirical potentials are such modelling tools for materials, since they can yield reasonable results. These potentials can also provide good explanations of the defect energies, surface energies, elastic properties, and mechanical aspects of oxides [15], fluorides [16] and many other materials [17,18]. Most of these potentials include Coulomb interactions, short-range pair interactions, and ionic polarization treated by the shell model of Dick and Overhauser [19]. The sum of the Coulomb terms, short-range interactions, and ionic polarization expresses the total energy for these potentials. If we presume that the electron cloud of an ion is simulated by a massless shell charge $q_{s}$ and the nucleus by a core of charge $q_{c}$, then the total charge becomes $q=q_{s}+q_{c}$. In the shell model interatomic potential approach, a harmonic force with spring constant $K$ couples the core and the shell of the ion. So, for modelling the short-range pair interactions acting between the shells, we can use a typical Buckingham potential, as presented in Equation (1):

$$
V_{i j}^{\text {Buckingham }}=A \exp \left(-\frac{r_{i j}}{\rho}\right)-C r_{i j}^{-6}
$$

The first part of Equation (1) indicates the Born-Mayer term, whereas the second part represents the Van der Waals energies. Further, in our work, we applied the original form of a mixed-type interatomic potential of Hamad et al. [20] for short-range interactions, which incorporates the Buckingham and Lennard-Jones 9-6 potentials form as in Equation (2):

$$
V_{i j}^{s h o r t}=A \exp \left(-\frac{r_{i j}}{\rho}\right)+B r_{i j}^{-9}-C r_{i j}^{-6}
$$


We also considered the semi-covalent nature of ZnS by using a three-body potential for S-Zn-S angel as in its original form [20] and expressed by Equation (3):

$$
V_{i j k}=\left(\frac{1}{2}\right) K_{T B}\left(\theta_{i j k}-\theta_{0}\right)^{2}
$$

In Equation (3), $\theta_{0}$ and $K_{T B}$ show the equilibrium constant angle between S-Zn-S and fitting constant of Hamad et al. [20], respectively. Lastly, sulphur anion polarizibility treated by the shell model of Reference [19] can be written as in Equation (4):

$$
V_{i j}^{\text {core-shell }}=\left(\frac{1}{2}\right) K r_{i j}^{2}
$$

where $r_{i j}$ describes the core-shell separation, and $K$ indicates the spring constant. Although extra details of presently employed potential and its parameterization procedure can be found in Reference [20], Table 1 lists the original potential parameters of Reference [20] used in our calculations.

Table 1. Mixed type theoretical interatomic potential used in this work. Parameters of the applied

\begin{tabular}{|c|c|c|c|c|}
\hline \multicolumn{5}{|c|}{ Mixed Potential Parameters from Ref. [20] } \\
\hline General potential & $\mathrm{A}(\mathrm{eV})$ & $\rho(\AA)$ & $\mathrm{B}\left(\mathrm{eV} . \AA^{9}\right)$ & $\mathrm{C}\left(\mathrm{eV} . \AA^{6}\right)$ \\
\hline $\mathrm{Zn}-\mathrm{S}$ & 213.20 & 0.475 & 664.35 & 10.54 \\
\hline S-S & 11,413 & 0.153 & 0.0 & 129.18 \\
\hline Three-body potential & $\theta_{0}$ (degree) & & $\mathrm{K}_{\mathrm{TB}}\left(\right.$ ev.rad $\left.^{-2}\right)$ & \\
\hline Zn core-S Shell-S Shell & 109. 47 & & 0.778 & \\
\hline Spring potential & & & $\mathrm{K}\left(\mathrm{eV} . \AA^{-2}\right)$ & \\
\hline S core-S Shell & 27.690 & & & \\
\hline Ion charges & Charge (e) & & & \\
\hline Zn core & 2.000 & & & \\
\hline$S$ core & 1.357 & & & \\
\hline S Shell & -3.357 & & & \\
\hline
\end{tabular}
potential were taken from its original reference [20].

All theoretical calculations in this work were carried out with the General Utility Lattice Program (GULP) 4.2 molecular dynamics code [21,22]. This versatile code allows the concerned structures to be optimized at constant pressure (all internal and cell variables are included) or at constant volume (unit cell remains frozen). To avoid the constraints, constant pressure optimization was applied to the geometry of WZ ZnS cell with the Newton-Raphson method based on the Hessian matrix calculated from the second derivatives. The cell geometry of WZ ZnS was assigned as $a=b=$ $3.811 \AA, c=6.234 \AA, \mathrm{u}=0.375$, and $\alpha=\beta=90^{\circ}$ and $\gamma=120^{\circ}$ with space group $P 6_{3} m c$. During the present geometry optimization calculations, the Hessian matrix was recursively updated with the BFGS [23-26] algorithm. After setting the necessities for the geometry optimization of WZ ZnS, we devised multiple runs at zero Kelvin temperature and checked the pressure ranges between 0 GPa and 12 GPa in steps of $3 \mathrm{GPa}$. Further, phonon and associated properties of WZ ZnS were also addressed after constant pressure geometry optimization calculations as a function of pressure within the quasiharmonic approximation under zero Kelvin temperature, as implemented in GULP code. It is possible to capture the phonon density of states (PDOS) and dispersions for a material after specifying a shrinking factor with GULP phonon computations. Further, phonons are described by calculating their values at points in reciprocal space within the first Brillouin zone of the given crystal. To achieve the Brillouin zone integration and obtain the PDOS, we have used a standard and reliable scheme developed by Monkhorts and Pack [27] with $8 \times 8 \times 8$-point mesh. 


\section{Results and Discussion}

Figure 1 indicates the density behavior of WZ ZnS under pressure. As is well known, the density of many materials exhibits clear increments under pressure $[6,15,17,18,28]$. This is also the case for WZ $\mathrm{ZnS}$ under pressure, as seen in Figure 1. The minimum and maximum values of the density of WZ ZnS was found to be $4.08 \mathrm{~g} / \mathrm{cm}^{3}$ and $4.59 \mathrm{~g} / \mathrm{cm}^{3}$ for $0 \mathrm{GPa}$ and $12 \mathrm{GPa}$, respectively, at zero temperature.

The elastic constants of materials not only provide precise and essential information about the materials, but also explain many mechanical and physical properties. Once the elastic constants are determined, one may get a deeper insight into the stability of the concerned material $[6,15,17,18,28]$. These constants can be also helpful to predict the properties of materials (i.e., interatomic bonding, equation of state, and phonon spectra). They also link to several thermodynamic parameters, such as the specific heat, thermal expansion, Debye temperature, Grüniesen parameter, etc. However, in general, elastic constants derived from the total energy calculations correspond to single-crystal elastic properties. So, the Voigt-Reuss-Hill approximation is a confident scheme for polycrystalline materials $[6,15,17,18,28]$. To obtain the accurate values of elastic constants and other analyzed parameters of WZ ZnS, the Voigt-Reuss-Hill values were taken into account for this research. For wurtzite crystal structures, five independent elastic constants exist as: $C_{11}, C_{12}, C_{13}, C_{33}$, and $C_{44}$ [28].

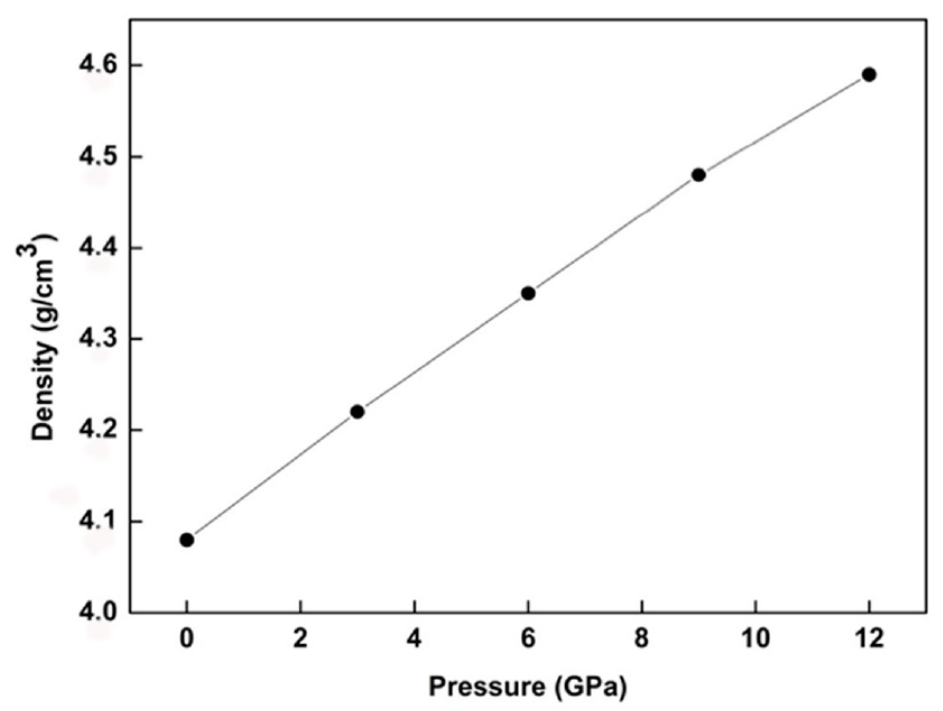

Figure 1. Density behavior of wurtzite (WZ) ZnS under pressure.

The plot in Figure 2 shows our results for $C_{11}, C_{12}, C_{13}, C_{33}$ and $C_{44}$ elastic constants of WZ ZnS under pressures between $0 \mathrm{GPa}$ and $12 \mathrm{GPa}$. All obtained elastic constants of WZ ZnS increase with the applied pressure except $C_{33}$ and $C_{44}$. Beyond these increments, a closer examination of Figure 2 reveals that the magnitudes of the elastic constants are in the range of: $C_{33}>C_{11}>C_{12}>C_{13}>C_{44}$. Both the range of elastic constants and slight decrement of $C_{44}$ constant under pressure mimic the DFT findings of Tan et al. [7]. However, our results for the ground state parameters of WZ ZnS are obviously better than those of Tan et al. [7] as well as Wright and Gale [11], and are much closer to the experimental results of Neumann et al. [29]. Table 2 gives a numerical comparison of present results for the elastic constants and other calculated quantities of $\mathrm{WZ} \mathrm{ZnS} \mathrm{with} \mathrm{some} \mathrm{previously} \mathrm{published}$ experimental and theoretical data. 


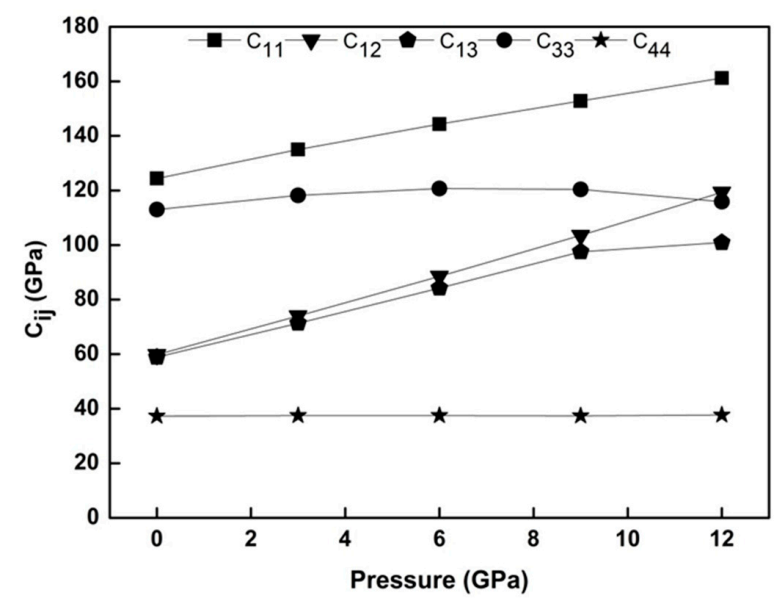

Figure 2. Elastic constants of WZ ZnS under pressure.

Table 2. Comparing our results with previous experimental and theoretical data for the considered parameters of WZ ZnS under zero pressure and temperature. * Bulk modulus experimental value was taken from Reference [30].

\begin{tabular}{|c|c|c|c|c|}
\hline \multirow{2}{*}{ Parameter } & \multirow{2}{*}{ Exp [29] } & \multirow{2}{*}{ Present } & \multicolumn{2}{|c|}{ Other Theoretical } \\
\hline & & & $\operatorname{Ref}[8]$ & $\operatorname{Ref}[14]$ \\
\hline $\mathrm{C}_{11}$ (GPa) & 124.2 & 124.4 & 115.6 & 135.4 \\
\hline $\mathrm{C}_{12}$ (GPa) & 60.1 & 59.8 & 48.9 & 65.8 \\
\hline $\mathrm{C}_{13}(\mathrm{GPa})$ & 45.5 & 58.9 & 37.1 & 51.6 \\
\hline $\mathrm{C}_{33}(\mathrm{GPa})$ & 140.0 & 113.0 & 132.5 & 160.7 \\
\hline $\mathrm{C}_{44}(\mathrm{GPa})$ & 28.6 & 37.3 & 27.2 & 32.4 \\
\hline B (GPa) & $75.8^{*}$ & 79.5 & 68.5 & 84.9 \\
\hline E (GPa) & & 75.3 & & \\
\hline G (GPa) & & 33.4 & & \\
\hline $\mathrm{V}_{\mathrm{S}}(\mathrm{km} / \mathrm{s})$ & & 2.86 & & \\
\hline $\mathrm{V}_{\mathrm{L}}(\mathrm{km} / \mathrm{s})$ & & 5.51 & & \\
\hline
\end{tabular}

From a stability outlook, the proverbial Born mechanical stability condition for a hexagonal structure also holds for wurtzite crystal, and must satisfy [28]:

$$
C_{44}>0, C_{11}>C_{12} \text { and }\left(C_{11}+2 C_{12}\right) C_{33}-2 C_{13}^{2}>0
$$

The present results for the obtained elastic constants of WZ ZnS satisfy the mechanical stability condition (Figure 2 and Table 2), and this result points out that $\mathrm{WZ} \mathrm{ZnS}$ is mechanically stable at $0 \mathrm{~K}$ temperature and 0 GPa pressure.

The bulk modulus $(B)$ is the only elastic constant of a material that conveys much information about the bonding strength. Moreover, it is a measure of the material's resistance to external deformation, and occurs in many formulae describing diverse mechanical-physical characteristics. The shear modulus $(G)$, however, portrays the resistance to shape change caused by a shearing force. In addition to $B$ and $G$, Young's modulus $(E)$ is the resistance to uniaxial tensions. These three distinct moduli $(B, G$, and $E)$ are other valuable parameters for identifying the mechanical properties of materials $[6,15,17,18,28]$.

Figure 3 represents the pressure behavior of $B, G$, and $E$ of WZ $\mathrm{ZnS}$ for the entire pressure range. From the prevalent physical definition of bulk modulus $(B=\Delta P / \Delta V)$ is expected an increment for $B$ because of its direct proportion to applied pressure. Hence, bulk modulus of WZ ZnS exemplifies a straight increment in Figure 3. Contrary to sharp increment in B, E and G moduli have slight decrements under pressure again similar to the DFT findings of Tan et al. [7]. Apart from the pressure behavior of these three moduli, Table 2 also summarizes another numerical comparison for $B$ with 
former experimental and theoretical data. The present result for the bulk modulus of WZ ZnS with 79.5 GPa approximately agrees with experimental values and is better than other DFT results, as seen in Table 2.

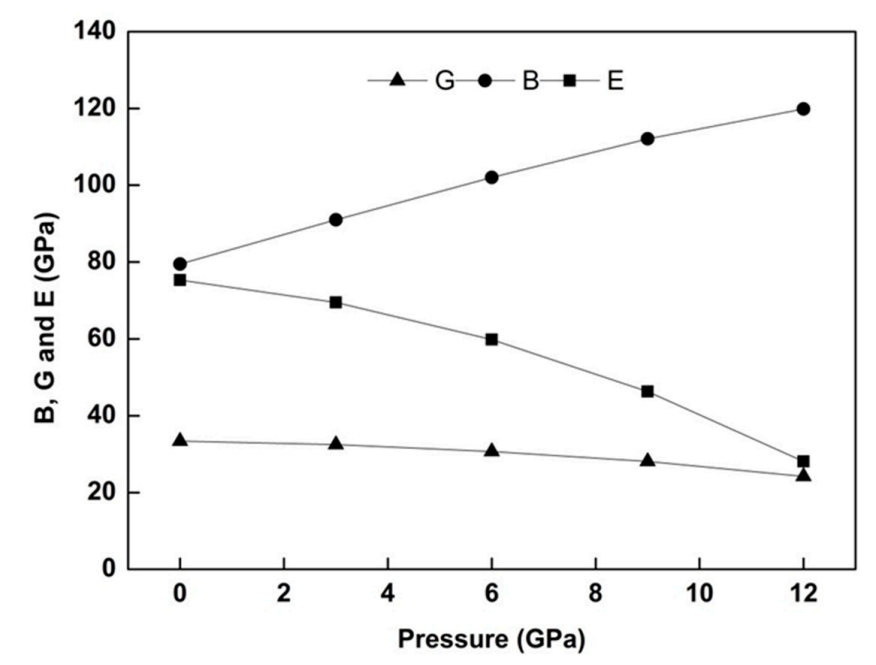

Figure 3. Elastic moduli (B, G, and E) behavior of WZ ZnS versus pressure.

The adjectives "brittle" and "ductile" signify the two distinct mechanical characters of solids when they are exposed to stress. Ductile and brittle features of materials play a key role during the manufacturing of materials $[6,15,17,18,28]$. So, we also evaluated the ductile (brittle) nature of WZ $\mathrm{ZnS}$ under pressure. In general, brittle materials are not deformable or are less deformable before fracture, whereas ductile materials are very deformable before fracture. At this point, the Pugh ratio is a determinative limit for the ductile (brittle) behavior of materials, and has popular use in literature. If the $B / G$ ratio is about 1.75 and higher, the material is accepted to be ductile; otherwise, the material becomes brittle $[6,15,17,18,28]$. After a careful evaluation, we determined $B / G$ values changing from 2.38 to 4.95 , with a monotonous increment between $\mathrm{P}=0 \mathrm{GPa}$ and $\mathrm{P}=12 \mathrm{GPa}$ at zero temperature, respectively, for WZ ZnS (as seen in Figure 4). So, this result manifests the ductile character of WZ ZnS in both ground state and under pressure. As another result, all values of the $\mathrm{B} / \mathrm{G}$ are higher than 1.75 and increase with pressure, which suggests that pressure can improve ductility (Figure 4).

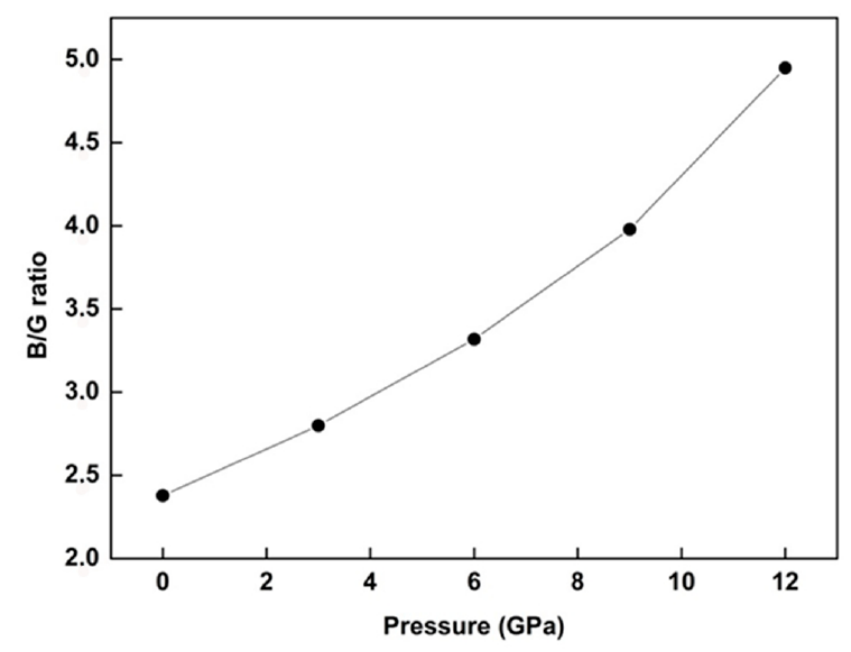

Figure 4. B/G ratio of $\mathrm{WZZ} Z \mathrm{ZnS}$ against pressure. 
In solids, low temperature ( $\mathrm{T}=0 \mathrm{~K}$ in our case) acoustic modes can trigger the vibrational excitations. Depending on this fact, two typical (longitudinal and shear) elastic waves exist. The velocity $V_{L}$ represents the longitudinal elastic wave velocity and $V_{S}$ denotes shear wave velocity $[6,15,17,18,28]$. Figure 5 shows the behavior of $V_{L}$ and $V_{S}$ elastic wave velocities for WZ $\mathrm{ZnS}$ as a function of pressure. $V_{L}$ has a substantial increment compared to $V_{S}$, which is the most observed circumstance for many materials.

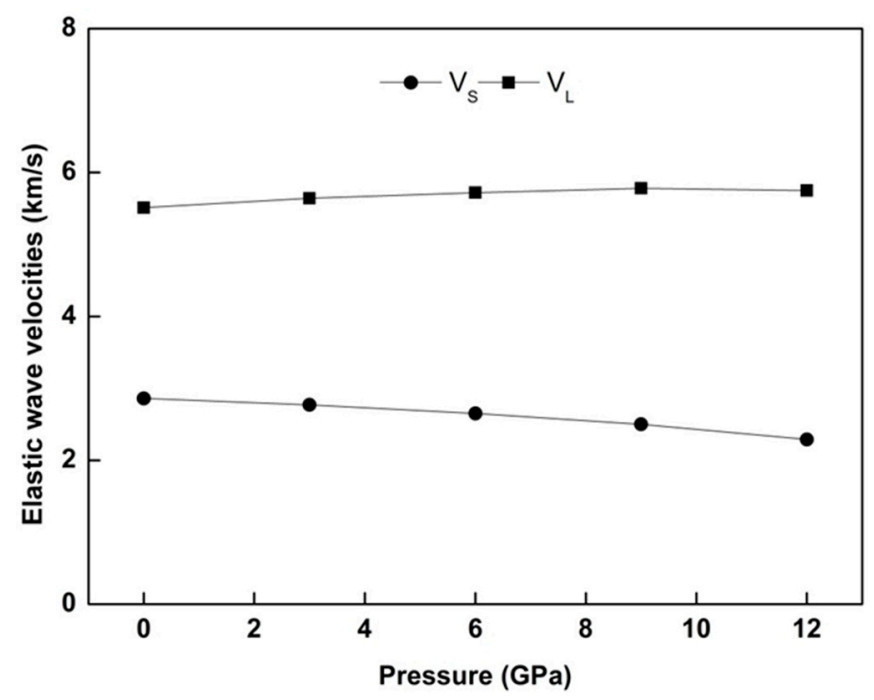

Figure 5. Behavior of longitudinal $\left(\mathrm{V}_{\mathrm{L}}\right)$ and shear wave $\left(\mathrm{V}_{\mathrm{S}}\right)$ velocities of $\mathrm{WZ} \mathrm{ZnS}$ under pressure.

Figure 6 shows the phonon dispersion of WZ ZnS along the chosen $\Gamma-\mathrm{A}$ path. In addition, Table 3 compares the numerical values of the zone-center ( $\Gamma$ points) phonon frequencies of this work with earlier experimental and theoretical data of WZ ZnS. As seen in Table 3, the agreement with experiment is quite good, especially for A1 (TO), E1 (TO) and A2 (TO), E2 (TO) phonon modes and competing with recent GGA and LDA DFT data of Reference [14].

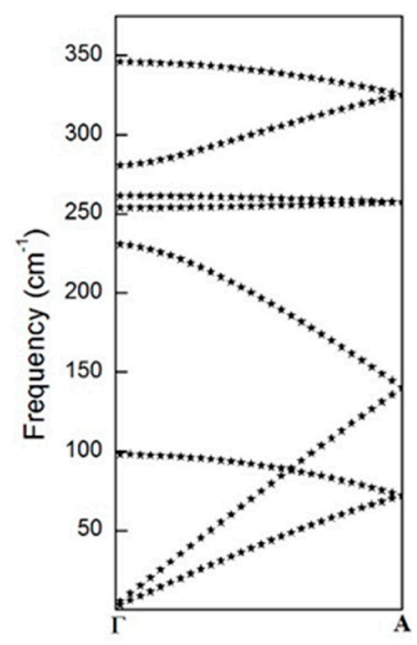

Figure 6. Phonon dispersion curve of $\mathrm{WZ} \mathrm{ZnS}$ at zero temperature along the chosen $\Gamma-\mathrm{A}$ path. 
Table 3. Comparing our results with previous experimental and theoretical data for the phonon frequencies $\left(\mathrm{cm}^{-1}\right)$ of $\mathrm{WZ} \mathrm{ZnS}$ under zero pressure and temperature.

\begin{tabular}{|c|c|c|c|c|}
\hline \multirow{2}{*}{ Symmetry } & \multirow{2}{*}{$\operatorname{Exp}[31]\left(\mathrm{cm}^{-1}\right)$} & \multirow{2}{*}{ Present $\left(\mathrm{cm}^{-1}\right)$} & \multicolumn{2}{|c|}{$\operatorname{Ref}[13]$} \\
\hline & & & GGA $\left(\mathrm{cm}^{-1}\right)$ & $\operatorname{LDA}\left(\mathrm{cm}^{-1}\right)$ \\
\hline $\mathrm{E}_{2}$ (low) & 72 & 98 & 68 & 69 \\
\hline $\mathrm{B}_{1}$ (low) & & 230 & 186 & 199 \\
\hline A1 (TO) & 273 & 279 & 257 & 290 \\
\hline E1 (TO) & 273 & 253 & 257 & 293 \\
\hline $\mathrm{E}_{2}$ (high) & 286 & 260 & 261 & 297 \\
\hline $\mathrm{B}_{1}$ (high) & & 279 & 318 & 347 \\
\hline A1 (LO) & 351 & 345 & 327 & 350 \\
\hline E1 (LO) & 351 & 352 & 324 & 349 \\
\hline
\end{tabular}

Further, we have focused on the ground state phonon density of states (PDOS) of WZ ZnS to clarify the contribution of the both elements ( $\mathrm{Zn}$ and $\mathrm{S}$ ) to the phonon properties of the compound. Figure 7 demonstrates the partial PDOS of WZ ZnS under zero pressure and temperature. As is apparent in Figure 7, the contribution of the Zn element to acoustic phonon modes is higher than S, whereas the opposite case is valid for the $S$ element due to its dominant contribution to the optical modes of the compound.

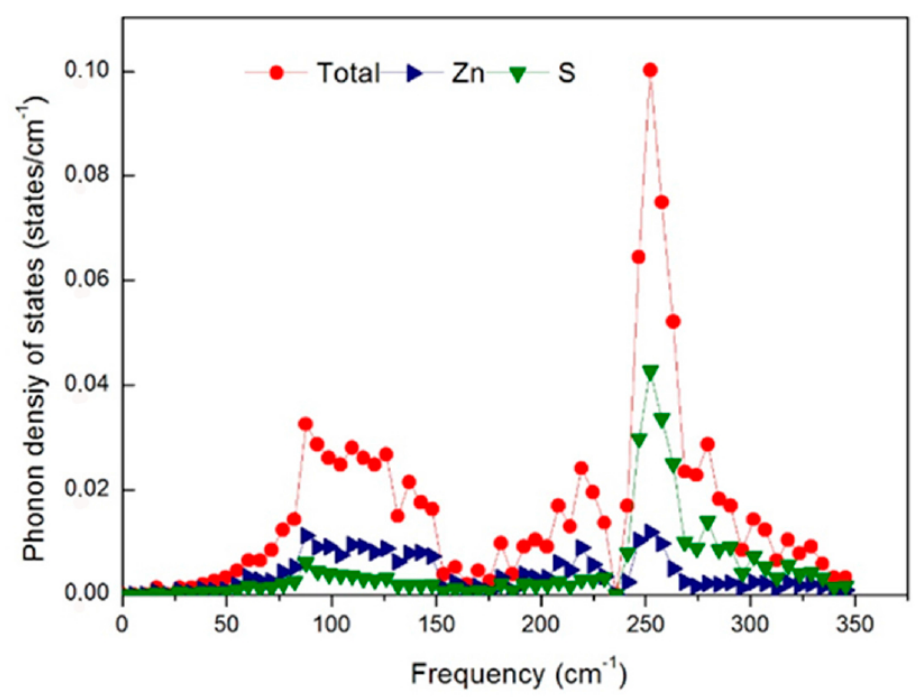

Figure 7. Partial and total phonon density of states of (PDOS) curve of WZ ZnS under zero temperature and pressure.

Figure 8 shows the phonon dispersion curves of WZ ZnS under different pressures. Each pressure value above $0 \mathrm{GPa}$ shifts the dispersion curves of WZ ZnS up slightly to the higher frequency values, as is clear in Figure 8. This behavior strictly originates from the atoms of WZ ZnS which are getting closer to each other under pressure, since they sit in steeper potential wells. The effect of pressure also causes the same behavior in the total density of states curves of WZ ZnS as shown in Figure 9. 


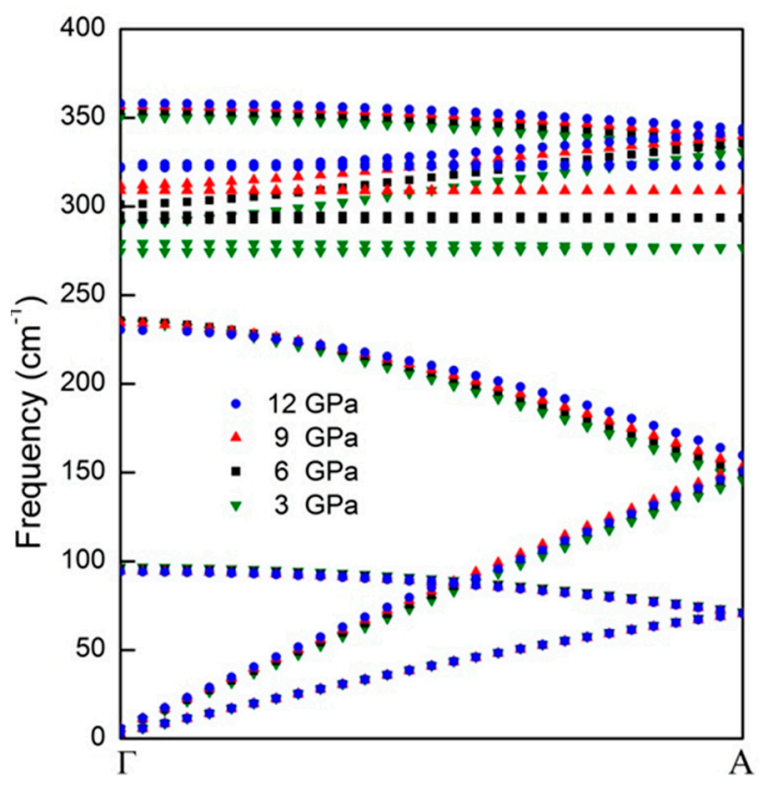

Figure 8. Phonon dispersion curves of $\mathrm{WZ} \mathrm{ZnS}$ at zero temperature along the chosen $\Gamma-\mathrm{A}$ path under different pressures.

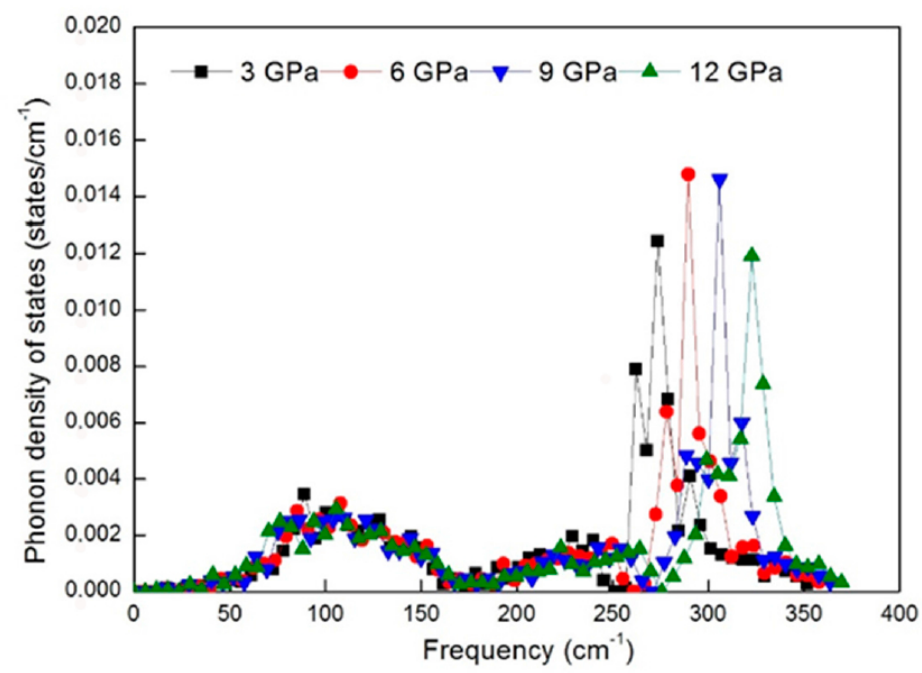

Figure 9. Total phonon density of states of (PDOS) curves of WZ ZnS under zero temperature with pressures $3 \mathrm{GPa}, 6 \mathrm{GPa}, 9 \mathrm{GPa}$, and $12 \mathrm{GPa}$.

Overall, the obtained results display a fair agreement with the experiments-in particular in elastic constants, bulk modulus, and phonon properties of WZ ZnS. Finally, the presented results for all considered quantities of $\mathrm{WZ} \mathrm{ZnS} \mathrm{through} \mathrm{this} \mathrm{research} \mathrm{are} \mathrm{not} \mathrm{only} \mathrm{consistent} \mathrm{with} \mathrm{experiments,}$ but also better than those of some published theoretical data.

\section{Conclusions}

In summary, we applied a mixed-type interatomic potential for the first time in conjunction with geometry optimization calculations to predict the high-pressure elastic, mechanical, and phonon properties of WZ ZnS. As our results demonstrate, the application of a mixed-type interatomic potential that is originally used for predicting the surface energies of zinc blende-type ZnS crystal structure well capture the elastic, mechanical and phonon features of WZ ZnS under pressure. From a quantitative evaluation, the obtained results of this work approximate the former experimental 
values-in particular for the elastic constants, bulk modulus, and phonon modes, and better than those of some other published data thanks to the sensitivity of the applied potential. The results of the present work may especially be helpful to future studies regarding the high-pressure elastic, mechanical, and other related properties of WZ ZnS.

Author Contributions: Melek Güler and Emre Güler conceived and designed the theoretical calculations; Melek Güler performed the all calculations; Melek Güler analyzed the all obtained data of work; both Melek Güler and Emre Güler wrote the paper.

Conflicts of Interest: The authors declare no conflicts of interest.

\section{References}

1. Bouhemadoua, A.; Allali, D.; Bin-Orman, S.; Muhammad Abud Al Safi, E.; Khenata, R.; Al-Douri, Y. Elastic and thermodynamic properties of the $\mathrm{SiB} 2 \mathrm{O} 4(\mathrm{~B}=\mathrm{Mg}, \mathrm{Zn}$ and $\mathrm{Cd})$ cubic spinels: An ab initio FP-LAPW study. Mater. Sci. Semicond. Process. 2015, 38, 192-202. [CrossRef]

2. Boudrifa, O.; Bouhemadou, A.; Guechi, N.; Bin-Omran, S.; Al-Douri, Y.; Khenata, R. First-principles prediction of the structural, elastic, thermodynamic, electronic and optical properties of Li4Sr3Ge2N6 quaternary nitride. J. Alloy. Compd. 2015, 618, 84-94. [CrossRef]

3. Boulechfar, R.; Meradji, H.; Chouahda, Z.; Ghemid, S.; Drablia, S.; Khenata, R. FP-LAPW investigation of the structural, electronic and thermodynamic properties of Al3Ta compound. Int. J. Mod. Phys. B 2015, 29, 1450244. [CrossRef]

4. Murtaza, G.; Gupta, S.K.; Seddik, T.; Khenata, R.; Alahmed, Z.A.; Ahmed, R.; Khachai, H.; Jha, P.K.; Bin Omran, S. Structural, electronic, optical and thermodynamic properties of cubic $\mathrm{REGa}_{3}(\mathrm{RE}=\mathrm{Sc}$ or $\mathrm{Lu})$ compounds: Ab initio study. J. Alloy. Compd. 2014, 597, 36-44. [CrossRef]

5. Zeng, Z.; Christos, S.G.; Baskoutas, S.; Bester, G. Excitonic optical properties of wurtzite ZnS quantum dots under pressure. J. Chem. Phys. 2015, 142, 114305. [CrossRef] [PubMed]

6. Güler, E.; Güler, M. A Theoretical Investigation of the Effect of Pressure on the Structural, Elastic and Mechanical Properties of ZnS Crystals. Braz. J. Phys. 2015, 45, 296-301. [CrossRef]

7. Tan, J.J.; Li, Y.; Ji, G.F. High-Pressure Phase Transitions and Thermodynamic Behaviors of Cadmium Sulfide. Acta Phys. Pol. A 2011, 120, 501-506. [CrossRef]

8. Bilge, M.; Kart, S.Ö.; Kart, H.H.; Çağın, T. Mechanical and electronical properties of ZnS under pressure. J. Ach. Mat. Man. Eng. 2008, 31, 29-34.

9. Grünwald, M.; Zayak, A.; Neaton, J.B.; Geissler, P.L. Transferable pair potentials for CdS and ZnS crystals. J. Chem. Phys. 2012, 136, 234111. [CrossRef] [PubMed]

10. Rong, C.X.; Hu, C.E.; Yeng, Z.Y.; Cai, L.C. First-Principles Calculations for Elastic Properties of ZnS under Pressure. Chin. Phys. Lett. 2008, 25, 1064. [CrossRef]

11. Wright, K.; Gale, J.D. Interatomic potentials for the simulation of the zinc-blende and wurtzite forms of ZnS and CdS: Bulk structure, properties, and phase stability. Phys. Rev. B 2004, 70, 035211. [CrossRef]

12. Cheng, Y.C.; Jin, C.Q.; Gao, F.; Wu, X.L.; Zhong, W.; Li, S.H.; Chu, P.K. Raman scattering study of zinc blende and wurtzite ZnS. J. App. Phys. 2009, 106, 123505. [CrossRef]

13. Yu, Y.; Fang, D.; Zhao, G.D.; Zheng, X.L. Ab initio Calculation of the thermodynamic properties of of wurtzite ZnS: Performance of the LDA and GGA. Chalcogenide Lett. 2014, 11, 619-628.

14. Ferahtia, S.; Saib, S.; Bouarissa, N.; Benyettou, S. Structural parameters, elastic properties and piezoelectric constants of wurtzite ZnS and ZnSe under pressure. Superlattices Microstruct. 2014, 67, 88-96. [CrossRef]

15. Güler, M.; Güler, E. High pressure phase transition and elastic behavior of europium oxide. J. Optoelectron. Adv. Mater. 2014, 16, 1222-1227.

16. Ayala, A.P. Atomistic simulations of the pressure-induced phase transitions in BaF2 crystals. J. Phys. Condens. Matter 2001, 13, 11741. [CrossRef]

17. Güler, E.; Güler, M. Phase transition and elasticity of gallium arsenide under pressure. Mater. Res. 2014, 17, 1268-1272. [CrossRef]

18. Güler, E.; Güler, M. Elastic and mechanical properties of hexagonal diamond under pressure. Appl. Phys. A 2015, 119, 721-726. [CrossRef] 
19. Dick, B.G.; Overhauser, A.W. Theory of the Dielectric Constants of Alkali Halide Crystals. Phys. Rev. 1958, 112, 90. [CrossRef]

20. Hamad, S.; Cristol, S.; Catlow, C.R.A. Surface Structures and Crystal Morphology of ZnS: Computational Study. J. Phys. Chem. B 2012, 106, 11002-11008. [CrossRef]

21. Gale, J.D. GULP: A computer program for the symmetry-adapted simulation of solids. J. Chem. Soc. Faraday Trans. 1997, 93, 629. [CrossRef]

22. Gale, J.D.; Rohl, A.L. The General Utility Lattice Program (GULP). Mol. Simul. 2003, 29, 291. [CrossRef]

23. Broyden, G.C. The Convergence of a Class of Double-rank Minimization Algorithms 1. General Considerations. J. Inst. Math. Appl. 1970, 6, 76. [CrossRef]

24. Fletcher, R. A new approach to variable metric algorithms. Comput. J. 1970, 13, 317. [CrossRef]

25. Goldfarb, D. A family of variable-metric methods derived by variational means. Math. Comput. 1970, 24, 23. [CrossRef]

26. Shanno, D.F. Conditioning of quasi-Newton methods for function minimization. Math. Comput. 1970, $24,647$. [CrossRef]

27. Monkhorst, H.J.; Pack, J.D. Special points for Brillouin-zone integrations. Phys. Rev. B 1976, 13, 5188. [CrossRef]

28. Güler, E.; Güler, M. High pressure elastic properties of wurtzite aluminum nitrate. Chin. J. Phys. 2014, 52, 1625-1635. [CrossRef]

29. Neumann, H. Lattice dynamics and related properties of $\mathrm{A}^{\mathrm{I}} \mathrm{B}^{\mathrm{III}}$ and $\mathrm{A}^{\mathrm{II}} \mathrm{B}^{\mathrm{IV}}$ compounds, I. Elastic constants. Cryst. Res. Technol. 2004, 39, 939-958. [CrossRef]

30. Hu, E.C.; Sun, L.L.; Zeng, Z.Y.; Chen, X.R. Pressure and Temperature Induced Phase Transition of ZnS from First-Principles Calculations. Chin. Phys. Lett. 2008, 25, 675-678.

31. Brafman, O.; Mitra, S.S. Raman Effect in Wurtzite- and Zinc-Blende-Type ZnS Single Crystals. Phys. Rev. 1968, 171, 931. [CrossRef]

(C) 2017 by the authors. Licensee MDPI, Basel, Switzerland. This article is an open access article distributed under the terms and conditions of the Creative Commons Attribution (CC BY) license (http:/ / creativecommons.org/licenses/by/4.0/). 